\title{
Low Shear Viscosity due to Anderson Localization
}

\author{
Ioannis Giannakis, ${ }^{1}$ Defu Hou, ${ }^{2,1}$ Jia-rong $\mathrm{Li}^{2}{ }^{2}$ and Hai-cang Ren ${ }^{1,2}$ \\ ${ }^{1}$ Physics Department, The Rockefeller University, 1230 York Avenue, New York, NY 10021-6399 \\ ${ }^{2}$ Institute of Particle Physics, Huazhong Normal University, Wuhan, 430079, China
}

\begin{abstract}
We study the Anderson Localization effect on the shear viscosity in a system with random medium by Kubo formula. We show that this effect can reduce the shear viscosity nonperturbatively. Then we discuss its possible implementation in heavy-ion collisions, where the created heavy bound states or other collective modes may play the role of the random scatterer underlying Anderson Localization effect.
\end{abstract}

PACS numbers:

One of intriguing properties regarding the newly identified quark-gluon plasma (QGP) created by relativistic heavy ion collisions [1, 2, 3, 4] is its near perfect hydrodynamical behavior. In particular the ratio of the shear viscosity to the entropy density, deduced from the elliptic flow measurement is very small $\eta / s \simeq 0.2$ [5]. The weak coupling calculation gives a larger ratio of order $5.12\left(g^{4} \ln \frac{1}{g}\right)^{-1}$ for $g<<1$ for three flavors of massless quark [6, 16], with $g$ the QCD running coupling constant, which becomes unreliable near $T_{c}$ where $g>1$. Non-perturbative effects are expected to play an instrumental role to explain the observed ratio.

An analytical calculation of QCD beyond perturbation theory is not available and the lattice simulation of transport coefficients [18] suffers from large error bars associated to the analytic continuation to the mass shell, and so far only for pure SU(3) Yang-Mills theory without quarks. A lower bound of the viscosity-entropy ratio was estimated using the uncertainty principle within the framework of kinetic theories $\frac{\eta}{s} \geq \frac{1}{12}[7]$. This bound is surprisingly close to that of the $\mathcal{N}=4$ supersymmetric Yang-Mills theory at large $N_{c}$ and large 't Hooft coupling following the conjectured AdS/CFT duality [8] $\frac{\eta}{s}=\frac{1}{4 \pi} \simeq 0.08$. The experimental result is rather close to these bounds and the underlying QGP seems strongly interacting [11].

Besides the lower bound of the viscosity-entropy ratio, there is little understanding of the physical mechanism that contributes to the suppression of the shear viscosity. One possibility is the existence of many zero energy bound states as was suggested in [9]. Another possibility is the anomalous viscosity in a weakly but an expanding QGP within the formalism of Boltzmann equation [10].

In this letter, we shall explore a new mechanism that may lead to a small viscosity in a system dominated by elastic scattering. This is the Anderson Localization (AL) effect studied in the context of condensed matter physics.

The prototype AL effect refers to the multiple scattering process of a wave propagating in a disordered medium where the individual scattering is elastic. Naively, one expects that the net scattered wave equals to the incoherent sum of individual ones but a closer look reals that this is not the case. The scattering amplitude following any path of multiple scattering is in phase with the one following its time reversed path when the scattering angle reaches 180 , independent of the location of the individual scattering events along the path. The elasticity or approximate one is required to maintain the phase relation between the two scattering waves. This coherence effect was first suggested by Anderson [12] and was verified experimentally by the intensity peak of the reflected light off the surface of an amorphous material with normal incidence [13] (the figure 1 of this paper provided an intuitive illustration of the AL effect). The field theoretic treatments of AL have been developed in the context of the electrical conductivity [14] in an amorphous metal and the energy transport of light wave in a random medium [15].

In what follows, we shall generalize the existing analysis for the Anderson Localization effect on the electrical conductivity and the energy diffusivity to the momentum transport coefficient, shear viscosity, by considering a massless scalar field in a random medium. The massless particles resembles the thermal partons in the QGP produced by RHIC. Then we assume that there are some random scatterers required for the AL effect, for instance, the heavy bound states produced in the heavy ion collisions, or reminiscent of collective modes of the initial state. Because of the crudeness of our model, our conclusion remains qualitative.

While the transport coefficients can be calculated by diagrammatic expansion of the exact Kubo formula, they are usually extracted from kinetic theory by the Chapman-Enskog approximation of the Boltzmann equation. It has been shown that the latter approach is equivalent to the ladder re-summation of the former one for self interacting scalar field and pure Yang-Mills field [17, 19]. The coherence effect underlying the Anderson Localization corresponds to a set of maximally crossed diagrams which is beyond the ladder ones, and therefore can not be obtained from the kinetic theory.

Let us consider a simple model of massless scalar field 
in a random medium. The Lagrangian reads

$$
\mathcal{L}=-\frac{1}{2} \frac{\partial \phi}{\partial x_{\mu}} \frac{\partial \phi}{\partial x_{\mu}}+\kappa \phi^{2},
$$

where

$$
\kappa=\sum_{j} u\left(\vec{r}-\vec{R}_{j}\right)
$$

Here $u\left(\vec{r}-\vec{R}_{j}\right)$ is the potential of an impurity at the random position $R_{j}$. The physics of $\mathrm{AL}$ is not sensitive to the details of the coupling. The Kubo formula of the shear viscosity is

$$
\eta=\frac{1}{10} \lim _{\omega \rightarrow 0} \lim _{\vec{q} \rightarrow 0} \operatorname{Im} \chi(\vec{q}, \omega)
$$

where $\chi(\vec{q}, \omega)$ is the Fourier transformation of the retarded two point Green function of the traceless stress tensor, $\pi_{i j}=\frac{\partial \phi}{\partial x_{i}} \frac{\partial \phi}{\partial x_{j}}-\frac{1}{3} \delta_{i j}(\nabla \phi)^{2}$, i. e.

$$
\chi(\vec{q}, \omega)=\frac{i}{\omega} \int d^{4} X e^{-i Q \cdot X}<\operatorname{Tr} \bar{\rho}\left[\pi_{i j}(X), \pi_{i j}(0)\right]>\theta(t) .
$$

where $\bar{\rho}=Z e^{-\beta H}$ is the the density operator with $\operatorname{Tr} \bar{\rho}=1$. The bracket $\langle\ldots\rangle$ stands for the average over the centers of individual impurity potentials, $\vec{R}_{j}$. The four vector notation $X=(\vec{r}, i t)$ for coordinate and $Q=(\vec{q}, i \omega)$ for momentum-energy are adapted. The two point function in eq.(4) can be manipulated with the CTP( closed time path ) formulation [20] or by more traditional means [14]. We find

$$
\begin{aligned}
\chi(\vec{q}, \omega) & =\frac{2}{\omega} \int \frac{d^{3} p}{(2 \pi)^{3}} \int \frac{d^{3} p^{\prime}}{(2 \pi)^{3}} I_{i j}(\vec{p}, \vec{q}) I_{i j}\left(\vec{p}^{\prime},-\vec{q}\right) \\
& \times \int_{-\infty}^{\infty} \frac{d p_{0}}{2 \pi i}\left\{\left[n\left(p_{0}^{-}\right)-n\left(p_{0}^{+}\right)\right] \Phi^{R A}\left(P, P^{\prime}, Q\right)\right. \\
& \left.-n\left(p_{0}^{-}\right) \Phi^{R R}\left(P, P^{\prime}, Q\right)+n\left(p_{0}^{+}\right) \Phi^{A A}\left(P, P^{\prime}, Q\right)\right\} \\
& =\chi^{R A}(\vec{q}, \omega)+\chi^{R R}(\vec{q}, \omega)+\chi^{A A}(\vec{q}, \omega)
\end{aligned}
$$

where $n\left(p_{0}\right)=\left(e^{\beta p_{0}}-1\right)^{-1}, p_{0}^{ \pm}=p_{0} \pm \frac{1}{2} \omega$ and $I_{i j}(\vec{p}, \vec{q})$ is the Fourier component of the derivative operators in $\pi_{i j}$. We have

$$
I_{i j}(\vec{p}, 0) I_{i j}\left(\vec{p}^{\prime}, 0\right)=\frac{2}{3} p^{2} p^{\prime 2} P_{2}\left(\hat{p} \cdot \hat{p}^{\prime}\right)
$$

with $P_{2}\left(\hat{p} \cdot \hat{p}^{\prime}\right)$ the second Legendre polynomial. The function $\Phi_{\alpha \beta}\left(P, P^{\prime}, Q\right)$ with $\alpha$ and $\beta$ equal to $\mathrm{R}$ (retarded) or $\mathrm{A}$ (advanced) can be expressed in terms the retarded and advanced full boson propagators

$$
G_{R(A)}(P)=\frac{i}{p_{0}^{2}-p^{2}-\Sigma_{R(A)}(P)}
$$

together with the $1 \mathrm{PI}$ vertex functions $\Gamma_{\alpha \beta}\left(P, P^{\prime}, Q\right)$, i.e.

$$
\begin{aligned}
& \Phi_{\alpha \beta}\left(P, P^{\prime}, Q\right)=G_{\alpha}\left(P_{+}\right) G_{\beta}\left(P_{-}\right) \times \\
& {\left[(2 \pi)^{3} \delta^{3}\left(\vec{p}-\vec{p}^{\prime}\right)-i \Gamma_{\alpha \beta}\left(P, P^{\prime}, Q\right) G_{\alpha}\left(P_{+}^{\prime}\right) G_{\beta}\left(P_{-}^{\prime}\right)\right],}
\end{aligned}
$$

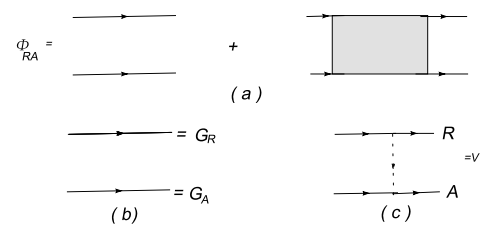

FIG. 1: . Diagrammatic representations

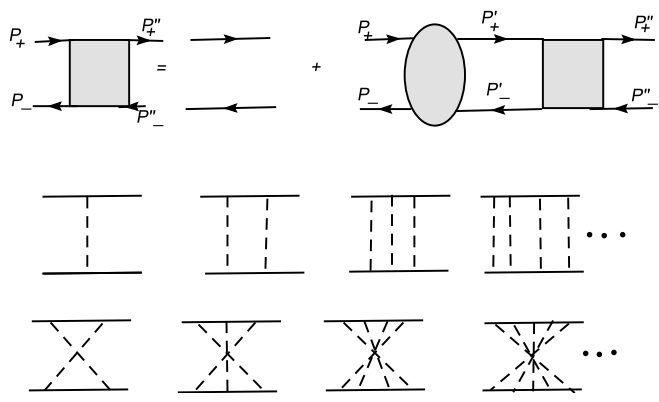

FIG. 2: . Schwinger Dyson equation and it's diagrammatic expansion

where $\Sigma_{R(A)}(P)$ is the retarded(advanced) self-energy function, $P_{ \pm}=P \pm \frac{1}{2} Q, P^{\prime}=\left(\vec{p}^{\prime}, i p_{0}\right)$ and $P^{\prime \prime}=\left(\vec{p}^{\prime \prime}, i p_{0}\right)$. We have $\Sigma_{R}(P)=\rho t_{\vec{p}, \vec{p}}\left(p_{0}\right)+O\left(\rho^{2}\right)$ where $t_{\vec{p}^{\prime}, \vec{p}}\left(p_{0}\right)$ denotes the $T$-matrix of the scattering by the potential $u(\vec{r})$ and $\rho$ is the density of the impurity centers. The Born approximation of $T$ matrix reads $t_{\vec{p}^{\prime}, \vec{p}}\left(p_{0}\right)=u_{\vec{p}}-\vec{p}$ with $u_{\vec{p}^{\prime}-\vec{p}}$ the Fourier transformation of $u(\vec{r})$. We have $\operatorname{Im} \Sigma_{R}(P)=-\rho p_{0} \sigma\left(p_{0}\right)+O\left(\rho^{2}\right)$ with $\sigma\left(p_{0}\right)$ the crosssection of the scattering by a single term of (2).

Since only $\Phi_{R A}\left(P, P^{\prime}, Q\right)$ contributes to the pinching singularity of the $p_{0}$-integration at weak coupling, we shall focus on it and see how the AL effect suppresses its contribution. The subscript $\mathrm{R}, \mathrm{A}$ of $\Gamma$ will be suppressed. The diagrammatic representation of $\Phi_{R A}$ together with the elements of the diagrams are displayed in Fig.1, where the upper(lower) solid line stands for the retarded(advanced) $\phi$-propagator and the dashed line denotes the impurity induced interaction. The bare vertex of Fig.1c reads $V=-2 i \pi \rho t_{\vec{p}_{+}^{\prime}, \vec{p}_{+}}\left(p_{0}^{+}\right) t_{\vec{p}_{-}^{\prime}, \vec{p}_{-}}^{*}\left(p_{0}^{-}\right) \delta\left(p_{0}^{\prime}-\right.$ $\left.p_{0}\right)$, from which the full vertex $\Gamma$ is generated. The energy delta function stems from the elasticity of the underlying scattering, which leaves the energy running along each solid line of $\Phi_{R A}$ conserved and all loop integrals inside $\Phi_{R A}$ over spatial momenta only. It follows from the Dyson-Schwinger equation for $\Gamma\left(P, P^{\prime}, Q\right)$, shown in Fig.2, that

$$
\begin{aligned}
& \Gamma\left(P, P^{\prime}, Q\right)=\tilde{\Gamma}\left(P, P^{\prime}, Q\right)- \\
& i \int \frac{d^{3} p^{\prime \prime}}{(2 \pi)^{3}} \tilde{\Gamma}\left(P, P^{\prime \prime}, Q\right) G_{R}\left(P_{+}^{\prime \prime}\right) G_{A}\left(P_{-}^{\prime \prime}\right) \Gamma\left(P^{\prime \prime}, P^{\prime}, Q \chi 9\right)
\end{aligned}
$$


which reduces to

$$
\begin{aligned}
& 2\left(p_{0} \omega+2 i \gamma p_{0}-\vec{p} \cdot \vec{q}\right) f\left(P, P^{\prime}, Q\right)=\tilde{\Gamma}\left(P, P^{\prime}, Q\right) \\
& -\int \frac{d^{3} p^{\prime \prime}}{(2 \pi)^{3}} \tilde{\Gamma}\left(P, P^{\prime \prime}, Q\right) \Delta G\left(P^{\prime \prime}, Q\right) f\left(P^{\prime \prime}, P^{\prime}, Q\right)(10)
\end{aligned}
$$

where $f\left(P, P^{\prime}, Q\right)=\Gamma\left(P, P^{\prime}, Q\right) /\left[2\left(p_{0} \omega+2 i \gamma p_{0}-\vec{p} \cdot \vec{q}\right)\right]$, $\Delta G(P, Q)=G_{R}\left(P_{+}\right)-G_{A}\left(P_{-}\right)$and $\tilde{\Gamma}\left(P, P^{\prime}, Q\right)$ is the 2PI part of $\Gamma\left(P, P^{\prime}, Q\right)$. The leading order of $\tilde{\Gamma}$ reads

$$
\tilde{\Gamma}_{0}=-i \rho t_{\vec{p}_{+}^{\prime}, \vec{p}_{+}}\left(p_{0}^{+}\right) t_{\vec{p}_{-}^{\prime}, \vec{p}_{-}}^{*}\left(p_{0}^{-}\right) .
$$

For $\left|\Sigma_{\alpha}(P)\right|<<p, \Delta G(P, Q)$ is sharply peaked at $p=p_{0}$ and we may approximate $\Sigma_{R}(P) \simeq-\Sigma_{A}(P) \simeq-2 i p_{0} \gamma$. Introducing the partial wave expansion

$$
\tilde{\Gamma}\left(P, P^{\prime}, Q\right)=\sum_{l}(2 l+1) c_{l}\left(p, p^{\prime}, \omega\right) P_{l}\left(\hat{p} \cdot \hat{p}^{\prime}\right)
$$

at $Q=(0, \omega)$ and that of $f\left(P, P^{\prime}, Q\right)$, an approximate solution to (10) at small $\omega$ and $\vec{q}$ can be obtained

$$
\Gamma\left(P, P^{\prime}, Q\right)=\frac{2 i \gamma c_{0}}{\omega+i D q^{2}}+4 \pi \gamma \sum_{l=2}^{\infty} \frac{(2 l+1) c_{l}}{\gamma_{l}} P_{l}\left(\hat{p} \cdot \hat{p}^{\prime}\right)
$$

where

$$
\gamma_{l}=\gamma+\frac{1}{4 i p_{0}} \int \frac{d^{3} \vec{p}^{\prime}}{(2 \pi)^{3}} \tilde{\Gamma}\left(P, P^{\prime}, Q\right) P_{l}\left(\hat{p} \cdot \hat{p}^{\prime}\right) \Delta G\left(P^{\prime}, Q\right)
$$

with the diffusion constant $D(\omega)=\frac{1}{6 \gamma_{1}}$ and the mass shell approximation $\Delta G(P, Q) \simeq 2 \pi \delta\left(p_{0}^{2}-p^{2}\right) \operatorname{sign}\left(p_{0}\right)$ has been employed. The emergence of the hydrodynamical pole ( or the absence of $\gamma_{0}$ ) is the consequence of the Ward identity [14]

$$
\Sigma_{R}(P)-\Sigma_{A}(P)=\int \frac{d^{3} \vec{p}_{1}}{(2 \pi)^{3}} \Delta G\left(P_{1}, Q\right) \tilde{\Gamma}\left(P_{1}, P, 0\right) .
$$

Inserting (13) into (8) and setting $Q=(0, \omega)$, we find

$$
\begin{aligned}
\Phi_{R A}= & -\frac{\pi^{2} \Delta G(P, Q) \delta\left(p-p^{\prime}\right)}{2 \gamma p_{0}^{3}}\left\{1-\frac{c_{0}}{4 \pi \omega}\right. \\
& \left.-\frac{\gamma}{8 i \pi} \sum_{l \geq 1} \frac{(2 l+1) P_{l}\left(\hat{p} \cdot \hat{p}^{\prime}\right)}{\gamma_{l}}\right\}
\end{aligned}
$$

The shear viscosity picks up $l=2$ partial wave of $\Phi\left(P, P^{\prime}, Q\right)$. It follows from (3), (5), (6), (16) and the mass shell approximation that

$$
\lim _{\omega \rightarrow 0} \chi_{R A}(0, \omega)=\frac{i \beta}{6 \pi^{2}} \int_{0}^{\infty} d p_{0} \frac{p_{0}^{4} e^{\beta p_{0}}}{\gamma_{2}\left(p_{0}\right)\left(e^{\beta p_{0}}-1\right)^{2}} .
$$

The sum of the ladder diagrams shown in the 2nd line of Fig. 2, that corresponds to the kinetic theory approach, can be obtained from the general solution (13) with $\tilde{\Gamma}$ of (12) and (14) replaced by its leading order (11).
The corresponding $\gamma_{l}$ 's will be denoted by $\gamma_{l}^{(0)}$. We have $c_{0}=-8 i \pi \gamma$ and

$$
\Gamma_{\text {ladder }}\left(P, P^{\prime}, Q\right)=\frac{16 i \pi \gamma^{2}}{i \omega-D_{0} q^{2}}+\ldots
$$

with the bare diffusion constant $D_{0}=\frac{1}{6 \gamma_{1}^{(0)}}$. The result of the shear viscosity following from the kinetic theory amounts to approximate $\gamma_{2}$ in the formula (17) by $\gamma_{2}^{(0)}$.

The AL effect induced by the backward coherent scattering is reflected in the maximally crossed diagrams shown in the third line of Fig. 2 which is a subset of 2PI diagrams for $\tilde{\Gamma}$. The sum of this set of diagrams, $U\left(P, P^{\prime}, Q\right)$ can be obtained from the that of ladder diagrams shown in the first line of Fig. 2 by reversing one of the rails (dropping the bare vertex ). We have

$$
U\left(P, P^{\prime}, Q\right)=\Gamma_{\text {ladder }}\left(P_{\text {rev }}, P_{\text {rev }}^{\prime}, Q_{\text {rev }}\right)
$$

where $P_{\text {rev }}=\left[\frac{1}{2}\left(\vec{p}-\vec{p}^{\prime}+\vec{q}\right), i p_{0}\right], P_{\text {rev }}^{\prime}=\left[\frac{1}{2}\left(\vec{p}^{\prime}-\vec{p}+\vec{q}\right), i p_{0}\right]$ and $Q_{\mathrm{rev}}=(\vec{p}+\vec{p}, i \omega)[14]$. We find that

$$
U\left(P, P^{\prime}, Q\right)=\frac{16 i \pi \gamma^{2}}{i \omega-D_{0}\left(\vec{p}+\vec{p}^{\prime}\right)^{2}}
$$

and $\tilde{\Gamma}\left(P, P^{\prime}, Q\right)=\tilde{\Gamma}_{0}+U\left(P, P^{\prime}, Q\right)+\ldots$. Therefore the maximally crossed diagrams renders the integrand of (14) to diverge in the backward direction, $\vec{p} \rightarrow-\vec{p}$, as $\omega \rightarrow 0$, and this singularity extends to all partial waves.

In space dimensions $d<3$, the backward singularity is sufficient to make $\gamma_{l}$ divergent at $\omega=0$ and thereby to make the dressed diffusion constant vanish there for arbitrarily weak scattering. But a critical strength of scattering is required for the purpose in $d=3$. A self consistent treatment of the backward singularity by summing up the most singular set of maximally crossed diagrams amounts to replaced the bare diffusion constant in the expression of $U\left(P, P^{\prime}, Q\right)$ with the dressed one, $D(\omega)$ [21]. Eq. (14) for $l=1$ becomes then a self-consistent equation for $D(\omega)$. Note that both $D_{0}$ and $D(\omega)$ depend on the energy $p_{0}$. Upon introducing a cutoff $k_{c}$ that restricts the momentum integration within the neighborhood of the backward scattering and approximating $\Delta G(P, Q)$ by its peak value 22], $\frac{1}{p_{0} \gamma}$, the self consistent equation for $D(\omega)$ becomes

$$
\frac{1}{D}=\frac{1}{D_{0}}+\frac{24 \pi \gamma^{2}}{p_{0}} \int_{\left|\vec{p}+\vec{p}^{\prime}\right|<k_{c}} \frac{d^{3} \vec{p}^{\prime}}{(2 \pi)^{3}} \frac{\hat{p} \cdot \hat{p}^{\prime}}{i \omega-D\left(\vec{p}+\vec{p}^{\prime}\right)^{2}} .
$$

which is of the same form as that in citezbsu. In terms of the localization length defined in [15], the limit

$$
\xi=\lim _{\omega \rightarrow 0} \sqrt{i \frac{D}{\omega}}
$$

the onset of $\mathrm{AL}$ is characterized by $\xi \neq 0$, which implies that $\lim _{\omega \rightarrow 0} D(\omega)=0$, and turns the hydrodynamical 
modes into localized ones via $i \omega-D\left(\vec{p}+\vec{p}^{\prime}\right)^{2} \rightarrow i \omega[1+$ $\left.\left(\vec{p}+\vec{p}^{\prime}\right)^{2} / \xi^{2}\right]$. Carrying out the integration of (21), we obtain the equation for the localization length,

$$
k_{c} \xi-\tan ^{-1}\left(k_{c} \xi\right)=\frac{\pi p_{0}^{2} \xi}{12 \gamma} .
$$

A real and nonzero solution for $\xi$ exists for

$$
\frac{\pi p_{0}^{2}}{12 \gamma^{2}}<1
$$

where we have set the cutoff $k_{c} \sim \gamma$. It follows from eq. (14) for $l=2$ ( with $D_{0}$ replaced by $D$ in $\Gamma$ ) that AL effect renders $\gamma_{2}\left(p_{0}\right) \rightarrow \infty$ when the condition (24) is met. While it is unlikely that the condition (24) holds for all $p_{0}$ ( because of the unitary bound that prevents $\gamma\left(p_{0}\right)$ from growing indefinitely with $\left.p_{0}\right)$, a finite domain of $p_{0}$ where AL takes place is quite possible. If the AL domain covers the peak of the function $p_{0}^{4} e^{\beta p_{0}} /\left(e^{\beta p_{0}}-1\right)^{2}$ in the integrad of the formula (17), a significant reduction of the shear viscosity is expected. In the parallel case of the Mie scattering of an electromagnetic wave by a random ensemble of metallic spheres studied in [15], AL does occur for $p_{0}$ greater than a critical value. But the upper bound of the AL domain was not reported there. But we have to admit that the mass-shell approximation employed to obtain the approximate solution (16) becomes marginal for $d=3$ because of the condition (24).

The self-consistent equation parallel to (21) for $d=2$ reveals that the localization length survives for an arbitrarily week coupling and for all $p_{0}$. No further conditions like (24) are required.

In conclusion, we have proposed a non-perturbative mechanism, the Anderson Localization effect, that may suppresses considerably the shear viscosity and other transport coefficients. The AL effect is caused by multiple coherent scattering in the system, which goes beyond the Boltzmann equation. It can be treated systematically in $d=2$ and self-consistently in $d=3$. Although the AL effect is discussed in a simple toy model in this letter, the physics of $\mathrm{AL}$ is more general. It only requires the individual scattering event to be sufficiently elastic. Regarding the QGP produced in RHIC, a potential source for AL is the scattering by the heavy bound states produced in collisions, whose masses could be as heavy as $2 \mathrm{GeV}$. If such states behaves like NR particles, the momentum transfer in a scattering event could exceed by far the energy transfer. Other non-equilibrium effect, such as the color gauge field produced in an expanding QGP [10] may also implement the elastic scattering necessary for AL. Much study is still needed to ascertain the relevance of AL to the low viscosity of sQGP and other RHIC phenomenology, say the energy losses process.
We would like to thank Professors A. Libchaber and Z. B. Su from whom we learnd the physics of Anderson Localization. We thank R. Pisarski, D. Rischke and T. Schäfer for interesting discussions . The work of I. G., H. C. R and D. F. H is supported in part by US Department of Energy under grants DE-FG02-91ER40651TASKB. The work of D. F. H. and H. C. R. is supported in part by NSFC under grant No. 10575043. The work of D. F. H. is also supported in part by Educational Committee under grants NCET-05-0675 and 704035.

[1] M. Gyulassy and L. McLerran, Nucl. Phys. A750(2005), 30.

[2] STAR collaboration, Phys. Rev. Lett. 90, 032301 (2003)

[3] PHENIX collaboration, Phys. Rev. Lett. 91, 182301 (2003)

[4] D.Molnar and P.Huovinen, Phys. Rev. Lett. 94, 012302 (2005) and references therein.

[5] D. Teaney, J. Phys. G30(2004) S1247; Phys. Rev. C 68, 034913 (2003) and references therein.

[6] P.Arnold, G.D.Moore and L.G.Yaffe, JHEP 0011, 001 (2000); 0305, 051 (2003)

[7] P. Danielewicz and M. Gyulassy, Phys. Rev. D31(1985) 53.

[8] G. Policastro, D. T. Son and A. O. Starinets, Phys. Rev. Lett., 87(2001) 081601; Alex Buchel and James T. Liu , Phys.Rev.Lett. 93, (2004) 090602.

[9] E. Shuryak, Prog. Part. Nucl. Phys. 53(2004) 273.

[10] M. Asakawa, S.A.Bass, and B.Mueller, Phys. Rev. Lett., 96(2006) 252301;

[11] Laszlo P. Csernai , J.I. Kapusta , Larry D. McLerran , Phys.Rev.Lett.97(2006)152303.

[12] P. Anderson, Phys. Rew. 109(1958), 1492.

[13] E. Akkermans, P. E. Wolf, and R. Maynard, Phy. Rev. Lett. 56, 1471 (1986).

[14] D. Vollhardt and P. Wolfle, Phys. Rev. B22(1980), 4666.

[15] K. Arya, Z. B. Su and J. L. Birman, Phys. Rev. Lett. $\mathbf{5 7}(1986) 2725$.

[16] G. Baym, H. Monien, C.J. Pethick and D.G. Ravenhall, Phy. Rev. Lett. 64, 1867 (1990); E. H. Heiselberg, D49, 4739 (1994).

[17] S. Jeon, Phys. Rev. D47, 4586 (1993); ibid. D52, 3591 (1995).

[18] A.Nakamura and S.Sakai, Phys. Rev. Lett. 94, 072305 (2005)

[19] M. E. Carrington, Hou Defu, and R. Kobes, Phys.Rev. D64, 025001 (2001); ibid, Phys.Lett. B523, 221 (2001); Hou, Defu, hep-ph/0501284.

[20] Z. B. Su, K. C. Chou, B. L. Hao and L. Yu, Phys. Rep. 118, (1985)1-131

[21] Jorgen Rammer, "Quantum transport theory", Persus books, reading, Massachusetts ,1998.

[22] The mass shell approximation has to be relaxed here because its application would lead to an artificial divergence of the integral over $\vec{p}^{\prime}$. 